\title{
DOENÇA DE CHAGAS NA AMAZÔNIA: I. REGISTRO DE OITO CASOS AUTÓCTONES EM MACAPÁ
}

\author{
Izabel de C. Rodrigues ${ }^{1}$, Adelson Almeida de Souza ${ }^{1}$, \\ René Terceros 2 e Sebastião Valente 1.
}

\begin{abstract}
Os autores descrevem 8 casos autóctones de doenca de Chagas ocorridos em Macapá. Os pacientes pertencem a duas familias, residindo em bairros distantes. Apresentaram sintomatologia comum como febre, cefaléia, mal-estargeral. A sorologia resultou positiva com titulos de IgM mais elevados que de $\mathrm{IgG}$ em $75 \%$ dos casos apresentados. Outros exames especificos para a doenca foram realizados. Os xenodiagnósticos foram positivos em $25 \%$ dos casos quando foi isolada a cepa de Trypanosoma cruzi. Os autores demonstram ainda que uma das formas infectadas do T. cruzi pertence ao zimodema 3 e sugerem a possibilidade de transmissão "per os".
\end{abstract}

Palavras-chaves: Trypanosoma cruzi. Doença de Chagas. Casos autóctones em Macapá. Zimodema.

Apesar da freqüência do Trypanosoma cruzi em reservatórios silvestres e triatomineos da floresta amazônica, nāo sāo freqüentes os casos em que o homem é acometido por infecção chagásica 46 .

Os primeiros estudos sobre doença de Chagas na Amazônia foram realizados por Rodrigues e Melo 10 , na região do Aurá, distante $10 \mathrm{~km}$ da cidade de Belém. Em estudos realizados no bairro de Canudos, Belém-PA, Shaw e cols ${ }^{11}$ registraram 4 casos autóctones da doença de Chagas. Outro caso, autóctone no Pará, foi constatado em julho de 1972 . O paciente residente em Belém apresentava sintomas caracteristicos da fase aguda da doença, cujo diagnóstico parasitológico resultou positivo 4 .

Quatro anos depois Lainson e cols ${ }^{4}$ registraram outro caso agudo em paciente natural de AbaetetubaPA, sem entretanto esclarecer sua etiologia. No ano seguinte era registrado em Belém, bairro de Sacramenta, mais um caso autóctone, confirmado por xenodiagnóstico 4 . Silveira e cols 12 relata o que seria o 9 ? caso clínico autoctone da doença em forma aguda ocorrido no Pará. Dorea ${ }^{3}$ faz o registro de mais um caso da doença no municipio de Santo Antonio do Tauá com sorologia positiva mas xenodiagnóstico negativo. No Territorio Federal do Amapá, o registro do primeiro caso da doença de Chagas foi feito por Nohmi ${ }^{9}$ em paciente natural da ilha de Bailique.

Incentivos financeiros do Wellcome Trust, London, da Fundação SESP - Instituto Evandro Chagas, Belém-PA e da Indústria e Comércio de Minérios (ICOMI), Macapá-AP. 1. Instituto Evandro Chagas (Fundaçāo SESP) - Av. Almirante Barroso, 492 - Caixa Postal: 1128 - 66050 - BelémPA

2. Hospital da Indústria e Comércio de Minérios (ICOMI)Porto Santana - 68900 - Macapá-AP.

Recebido para publicação: 15/04/88.
Neste trabalho os autores registraram o aparecimento, quase simultâneo, de 8 pacientes portadores da doença de Chagas, em Macapá-AP, evidenciando a autoctonia dos casos.

\section{MATERIAL E MÉTODOS}

Logo que soubemos dos casos ocorridos em Macapá programamos uma viagem com objetivo de esclarecer os fatos.

Informaçôes sobre dados clínicos, foram obtidas diretamente do Diretor do Hospital da ICOMI onde 2 pacientes: RSM ( 4 anos) e NSM ( 35 anos) estiveram internados. Deixamos de publicar os dados clínicos por falta de acesso aos mesmos.

No Instituto Evandro Chagas foram realizados os seguintes exames:

a) exame de sangue direto a fresco além de gota espêssa e estregaço corado pela Giemsa;

b) xenodiagnóstico utilizando-se 5 ninfas de III ou IV estágio das espécies Rhodnius prolixus ou Triatoma infestans dependendo da disponibilidade. Trinta dias depois da alimentação dos triatomineos foi realizado $o$ exame do conteúdo intestinal. Quando positivo, o material era inoculado em camundongos albinos (IP) ou semeado em meio Hoff;

c) ainda na tentativa de isolar a cêpa infectante foram executadas hemocultura, em meio Hoff, e inoculação em camundongos albinos (IP) com sangue periférico dos pacientes. No 20 ? dia, após semeadura em cultura ou inoculação em animais, eram realizadas as primeiras observaçōes;

d) titulação de anticorpos IgG e IgM, através de imunofluorescência indireta, no soro de todos os pacientes. $\mathrm{O}$ antígeno utilizado foi preparado com formas de cultura da cepa "Y" de T. cruzi (Instituto de 
Rodrigues IC, Souza AA, Terceros R, Valente S. Doença de Chagas na Amazônia: I. Registro de oito casos autóctones em Macapá. Revista da Sociedade Brasileira de Medicina Tropical 21: 193-197, Out-Dez, 1988

Medicina Tropical de São Paulo) e o conjugado antiIgG ou anti-IgM humanas da Wellcome Reagents Limited (Wellcome Research Laboratories Beckenham - England BR 3.3 PBS);

e) eletroforese em gel de amido para caracterização da cepa isolada. Foram ensaiadas 6 enzimas: aspartatoamino-transferase (E.C.2.6.1.1., ASAT); alaninaamino-transferase (E.C.2.6.1.2., ALAT); fosgoglucomutase (E.C.5.3.1.9., GPI); malato-dehidrodenase (oxalacetato-descarbosilase) (NADP +) (E.C.1.1.1. 40., ME) e glucose-6-fosfato dehidrogenase (E.C.1.1. 1.49., G6PD)6. Amostras de padrões clonados de zimodemas 1,2 e $3(Z 1, Z 2$ e Z3) foram incluidas em todas as placas de gel e os resultados interpretados através de simples comparação visual.

\section{RESULTADOS}

Apresentação dos casos: os pacientes, JLM, NSM, RSM e JCS, apresentaram sinais clinicos mais evidenciados da doença e por isso são descritos com maiores detalhes.

JLM, 40 anos, masculino, nasceu em AporemaAP, residindo desde a idade de 16 anos em Macapá. Exerce atividade no serviço de construção da estrada de ferro que liga Porto Santana à Serra do Navio (ICOMI). Em 26 de outubro de 1984 foi atendido no Hospital da ICOMI apresentando edema bipalpebral unilateral esquerdo. Cerca de um mês depois apresentava febre elevada, cansaço ao menor esforço fisico e mal-estar geral.

NSM, 35 anos, feminino, natural de Macapá, sempre residiu no bairro de Santa Rita. Em 28 de outubro foi à consulta no Hospital da ECOMI com queixas de cefaléia, febre continua, cansaço, edema bipalpebral bilateral e de membros inferiores. Esteve internada durante 7 dias submetendo-se a exames clínicos e laboratoriais.

RSM, 4 anos, feminino, natural de Macapá, bairro de Santa Rita. Foi conduzido ao Hospital da ICOMI em 28 de outubro de 1984 com queixas de febre continua, cefaléia, calafrio, mal-estar geral. Durante o periodo de internamento ( 10 dias) observou-se evolução do quadro clinico para miocardite e insuficiência cardíaca congestiva. Pertencentes a essa mesma familia existem ainda: RSM (10 anos) masculino; MSM (8 anos), masculino e RSM (6 anos) feminino. Apesar de apresentarem sintomas comuns, não apresentaram sinais clínicos caracteristicos nem foi observada a evolução da doença. Foram igualmente submetidos a exames laboratoriais especificos cujos resultados estão na Tabela 1 .

JCS, 2 anos e 5 meses, masculino, nasceu em Macapá, residindo no bairro do Pacoval. Foi conduzido ao Laboratório Central de Saúde Pública de Macapá (LCSPM) com os seguintes sintomas: febre alta continua e diarréia. Sua genitora, APCS, 26 anos, natural de Macapá, embora assintomática no momento em que chegou ao IBC foi também submetida a exames especificos.

$\mathrm{Na}$ Tabela 1, estão demonstrados os resultados obtidos dos exames processados no Instituto Evandro Chagas, Belém-PA.

Apenas no caso do menor JCS o exame de sangue a fresco (26.12.84) foi positivo, mostrando formas tripomastigotas de $T$. cruzi, confirmando as

Tabela 1 - Resultados de exames realizados em 8 pacientes suspeitos de portar doença de Chagas, originários de Macapá-AP.

\begin{tabular}{|c|c|c|c|c|c|c|c|c|c|c|}
\hline \multirow[t]{2}{*}{ Paciente } & \multirow[t]{2}{*}{ Sexo } & \multirow[t]{2}{*}{ Idade } & \multicolumn{2}{|c|}{ Pesquisa de $T$. cruzi } & \multirow[b]{2}{*}{$\begin{array}{l}\text { Gota } \\
\text { espessa }\end{array}$} & \multicolumn{2}{|c|}{ Hemocultura } & \multirow{2}{*}{$\begin{array}{c}\text { Xeno- } \\
\text { diag- } \\
\text { nóstico }\end{array}$} & \multicolumn{2}{|c|}{$\begin{array}{l}\text { Imunofluo- } \\
\text { rescència }\end{array}$} \\
\hline & & & $\begin{array}{l}\text { Exame direto } \\
\text { (a fresco) }\end{array}$ & $\begin{array}{l}\text { Esfre- } \\
\text { gaço }\end{array}$ & & $\begin{array}{l}30 \circ \\
\text { dia }\end{array}$ & $\begin{array}{l}60 \% \\
\text { dia }\end{array}$ & & $\begin{array}{l}\text { Anti- } \\
\text { IgG }\end{array}$ & $\begin{array}{l}\text { Anti- } \\
\text { IgM }\end{array}$ \\
\hline JLM & $\mathbf{M}$ & $40 a$. & - & - & - & + & & & $1: 640$ & $1: 160$ \\
\hline NSM* & $\mathrm{F}$ & $35 a$ & - & - & - & - & - & - & $1: 20$ & 1.320 \\
\hline RSM & $\mathbf{M}$ & $10 \mathrm{a}$ & - & - & - & - & - & - & Neg. & $1: 160$ \\
\hline MSM & $\mathbf{M}$ & $8 a$ & - & - & - & - & - & - & $1: 20$ & $1: 320$ \\
\hline RSM & $\mathrm{F}$ & $6 a$ & - & - & - & - & - & - & $1: 320$ & $1: 320$ \\
\hline $\mathrm{RSM}^{*}$ & $\mathrm{~F}$ & $4 a$ & - & - & - & - & $=$ & - & $1: 80$ & $1: 320$ \\
\hline $\mathrm{JCS}$ & $\mathbf{M}$ & $2 \mathrm{a} 5 \mathrm{~m}$ & + & - & - & + & $t$ & + & $1: 80$ & $1: 320$ \\
\hline APCS & $\mathrm{F}$ & $26 a$ & - & - & - & - & - & - & $1: 20$ & $1: 160$ \\
\hline
\end{tabular}

* Positivos para formas tripomastigotas morfologicamente semelhantes ao T. Cruzi, em exame realizado no Hospital da ICOMI, Santana, Macapa-AP. 
Rodrigues IC, Souza AA, Terceros R, Valente S. Doença de Chagas na Amazónia. I. Registro de oito casos autóctones em Macapá. Revista da Sociedade Brasileira de Medicina Tropical 21: 193-197, Out-Dez, 1988

suspeitas levantadas ainda no LCSPM onde foi realizada a hemoscopia.

Os exames em gota espessa e esfregaço resultaram $100 \%$ negativos. As pacientes NSM ( 35 anos) e RSM (4 anos), enquanto hospitalizadas, foram submetidas aos exames referidos sendo detectadas formas tripomastigotas morfologicamente semelhantes ao $T$. cruzi (comunicação pessoal), observação realizada antes de nossa equipe chegar ao local.

As culturas em meio Hoff obtidas a partir do sangue dos pacientes foram examinadas 30 e 60 dias após semeadura. No $30 \%$ dia, as culturas de JLM e JCS foram positivas para formas epimastigotas de $T$. cruzi mesmo após 60 dias.

Os animais inoculados com sangue periférico dos pacientes foram examinados semanalmente durante 30 dias sendo negativas $100 \%$ das preparações observadas.

Os testes de imunofluorescência indireta resultaram positivos para IgG em $87,5 \%$ dos casos variando os títulos entre 1:20 e 1:640. Os testes para IgM resultaram $100 \%$ positivos observando-se titulos entre 1:160 e 1:320. Em 62,5\% dos casos os titulos de IgM
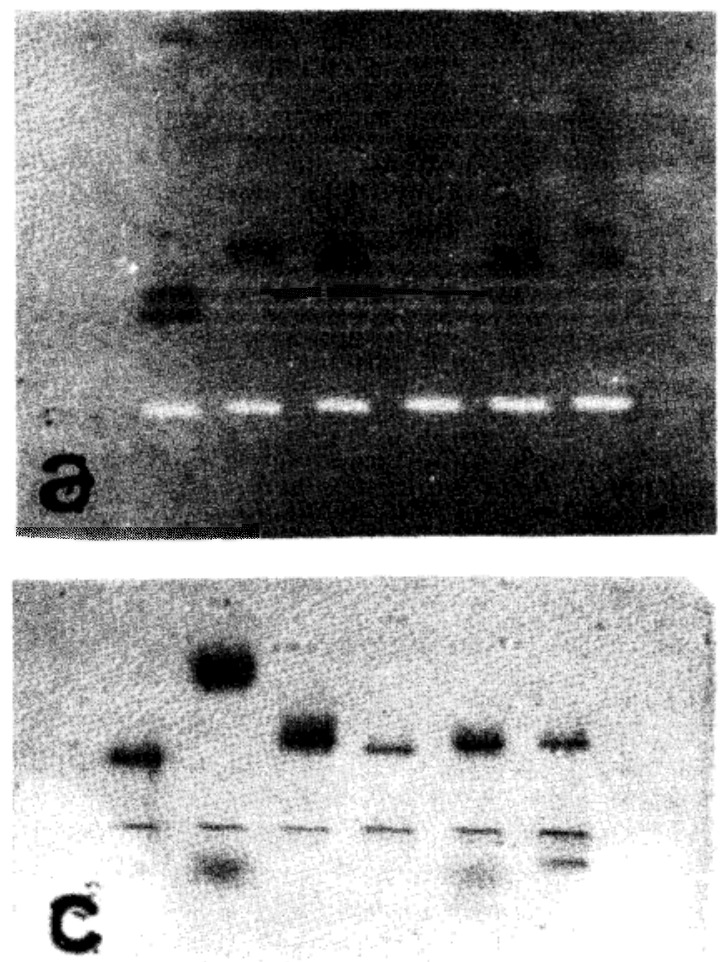

Figura 1 - Placas de eletroforese contendo a amostra de JCS, configurando o Zimodema 3. A amostra isolada foi comparada com os padrões de T. cruzi $e \mathrm{~T}$. rangeli. $A s$ enzimas mostradas são: a) ASAT; b) ALAT; c) GPI e d) PGM.

Em cada placa as amostras de Trypanosoma mostram apresentaram-se mais elevados do que aqueles obtidos para IgG.

O exame do conteúdo intestinal dos triatomineos mostrou-se positivo em 2 casos: JCS e JLM. As formas tripomastigotas metacíclicas e epimastigotas observadas foram cultivadas também em meio Hoff.

A única mostra que conseguimos isolar e preparar para caracterização bioquímica foi a do caso JCS. Na figura 1, podemos observar amostras de $T$. cruzi que, se considerados da esquerda para direita, correspondem aos padrões clonados de zimodemas 1,2 e 3 de $T$. cruzi è à amostra isolada JCS. Os resultados obtidos mostraram que a combinação de padrões eletroforéticos, de pelo menos 4 enzimas selecionadas para comparação de isoenzimas da amostra de $T$. cruzi isolada JCS, permitiu identificá-la como zimodema 3.

As primeiras buscas aos triatomineos nas residências e arredores, durante 3 dias e 3 noites, resultaram negativas bem como a captura de animais que pudessem servir como reservatório do parasita.

Estudos mais amplos sobre a epidemiologia desses casos estão sendo realizados e serão apresentados oportunamente.
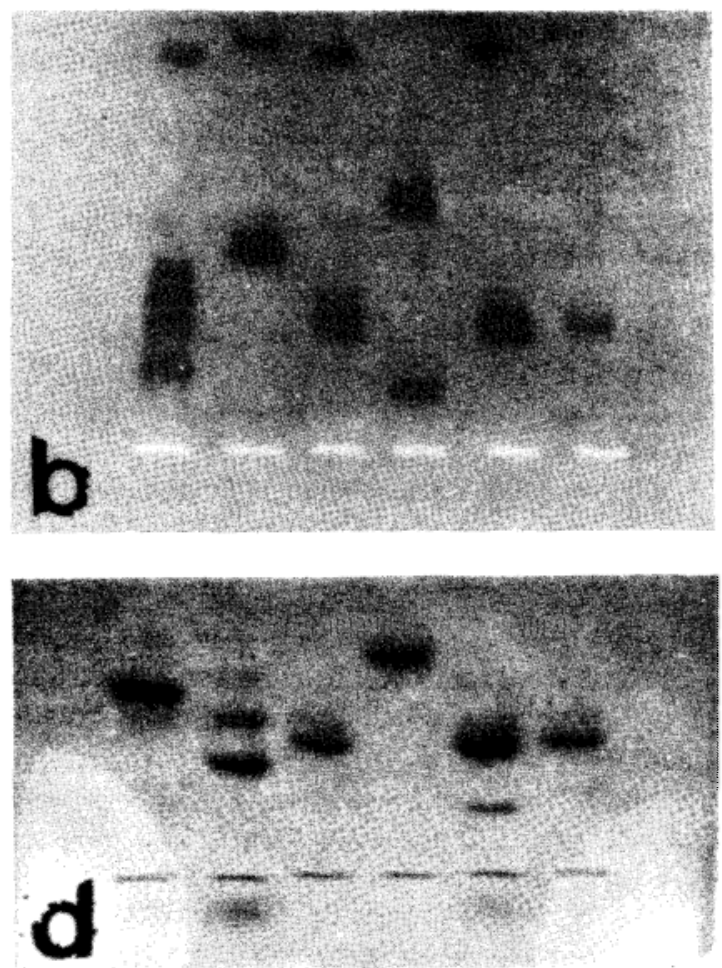

(da esquerda para a direita): 1 -X10 clone l, padrào de $\angle 1$ : 2 - Esmeraldo clone 3, padrão de $Z_{2} ; 3$ - CAN III clone I, padrão de $Z_{3 ;} 4-R 1625$, Trypanosoma rangeli do homem, El Salvador; 5-JCS, amostra do paciente e 6-Caixa 1016, Camundongo inoculado com fezes de XE 2239 (JCS). 


\section{DISCUSSÃO}

$\mathrm{Na}$ tentativa de esclarecer a etiologia dos casos, pacientes e parentes próximos foram cuidadosamente interrogados em relação ao afastamento do local de origem para outras regiōes do pais.

Por ocasião do exame fisico dos pacientes, não foi detectada nenhuma lesão que sugerisse "porta de entrada" do parasita. Soubemos que os pacientes JLM e NSM apresentaram, em fins de outubro de 1984, edema bipalpebral unilateral esquerdo e bilateral, respectivamente, tendo sido diagnosticada conjuntivite. Não podemos afirmar que se tratava de sinal de Romaña, pois já haviam se passado quase 3 meses quando lá chegamos. O sinal de Romaña é raro em nossa região, tendo sido observado uma única vez por Dorea $^{\mathfrak{3}}$ num caso agudo no interior do Pará.

A negatividade dos xenodiagnósticos de RSM (4 anos) e NSM ( 35 anos), justifica-se pelo inicio do tratamento especifico para a doença no dia imediato ao aparecimento do $T$. cruzi no sangue periférico. Igual justificativa damos à negatividade das culturas em meio Hoff e inoculação em animais com material dessas pacientes. A rápida evolução do quadro clínico, do caso RSM (4 anos), para miocardite e insuficiência cardiaca congestiva, contribui para comparação da instalação da doença.

Os títulos de IgG e IgM obtidos são compativeis com a instalação da fase aguda da doença. Inquéritos sorológicos quantitativos sobre infecção chagásica na Amazônia são escassos na literatura. Camargo ${ }^{1}$ realizou inquérito sorológico nacional para estimar a prevalência da infecção chagásica no Brasil mostrando resultados qualitativos, obtidos no Acre e Amazonas, sendo dificil estabelecer comparação com nossos resultados.

Do ponto de vista bioquimico, embora as cepas de $T$ cruzi, que causam a doença de Chagas nas zonas endêmicas, sejam enzimaticamente diferentes das cepas da Amazônia, essas últimas parecem ser igualmente virulentas na maioria dos casos em Belém ${ }^{4}$, como no caso de JCS, cuja caracterização bioquímica mostrou zimodema 3 como responsável pelo quadro agudo instalado.

Resultados obtidos anteriormente mostraram 4 casos autóctones de doença de Chagas, em Canudos, bairro de Belem ${ }^{11}$, sendo todos infectados com zimodema 36 , bem como um caso agudo no interior do Pará descrito por Dorea ${ }^{3}$. Ainda esse autor conseguiu capturar um exemplar da espécie Panstrongylus geniculatus no interior da casa do paciente. Essa espécie pode, na maioria das vezes, ser portadora de $T$. cruzi - zimodema 36 . Entretanto, não nos foi possível sequer detectar a presença de qualquer espécie de triatomineo durante as buscas efetuadas nas residências.
A falta de dados epidemiológicos dificulta o esclarecimento do mecanismo de transmissão nos casos observados em Macapá.

A transmissão por via oral poderia ser uma hipótese para explicar o desenvolvimento simultâneo da forma aguda da doença, em pessoas pertencentes às duas familias, em cujas residências não havia evidência de vetores triatomineos que pudessem ser incriminados na transmissão.

No primeiro registro de caso autóctone de doença de Chagas no Pará feito por Shaw e cols ${ }^{11}$, também não se conseguiu esclarecer o modo de transmissão.

Lainson e cols 5 infectaram $100 \%$ de 30 camundongos alimentados com diferentes tipos de comidas contaminadas por T. cruzi, zimodema 3.

Di Primio 2 descreve surto epidêmico de doença de Chagas em Teutônia-RS, possivelmente por transmissão oral.

O paciente JLM, operário da estrada-de-ferro que liga Santana à Serra do Navio, afirma ser costume trazer frutas e raízes para casa aos finais de semana quando retorna do acampamento onde trabalha. Não podemos descartar a hipótese de que algum exemplar de triatomíneo tenha sido, dessa maneira, conduzido para o interior do domicilio, apesar de nossas buscas aos insetos terem resultado negativas. Por outro lado devemos considerar que, quando nossa equipe chegou ao local já eram decorridos 2 meses.

Finalmente, existe ainda a possibilidade da transmissão através de formas infectantes de $T$. cruzi na urina de marsupiais (mucuras), costumeiros habitantes de quintais e cozinhas de residências. Devido ao hábito noturno desses animais, há a possibilidade de contaminação de alguma fonte de alimentação.

Face às observações registradas, podemos apenas concluir, no momento, que devido à ausência de medidas de controle, por parte das autoridades competentes, a instalação da endemicidade da doença de Chagas na Amazônia pode tornar-se realidade.

\section{SUMMARY}

The present paper describes 8 autochthonous cases of Chagas' disease in Macapa City, Federal Territory of Amapa, Brazil. The patients belonged to two different families and lived in different areas of the city. All the patients had general symptoms such as headache, fever and malaise. The serology was positive for $\mathrm{T}$. cruzi. The titres of IgM were higher than $\operatorname{IgG}$ in 6 of the cases. Other specific tests for Chagas' disease were carried out. Xenodiagnosis was positive in 2 of the cases and the T. cruzi strains were isolated in this instance. The authors had also demonstrated that one of the isolates of $\mathrm{T}$. cruzi was zymodeme 3 and suggest that transmission "per os" 
Rodrigues IC, Souza AA, Terceros R, Valente S. Doença de Chagas na Amazônia: I. Registro de oito casos autóctones em Macapá. Revista da Sociedade Brasileira de Medicina Tropical 21: 193-197, Out-Dez, 1988

most likely accounted for concomitant acute cases within the same family.

Key-words: Trypanosoma cruzi. Chagas' disease. Autochthonous cases in Macapa. Zymodeme.

\section{AGRADECIMENTOS}

Os autores expressam seus agradecimentos aos Drs. JJ. Shaw e R. Lainson pela orientação, estimulo e revisão do manuscrito. À Sra. Maria José Ferreira pela realização dos testes sorológicos. Ao Dr. José Evaldo Lima, Diretor da SUCAM de Macapá, e guardas pertencentes à sua equipe. À Dra. Elza Lopes da Silva, diretora do Laboratorio Central de Saúde Pública, Macapá, pelo encaminhamento de um dos casos apresentados. À Sra. Maria das Graças Silva pelo serviço datilográfico. Aos Srs. Aluízio Figueiredo pelo serviço fotográfico, Francisco Gomes e Raimundo Nivaldo de Almeida pelo auxílio na captura dos animais.

\section{REFERĖNCIAS BIBLIOGRÁFICAS}

1. Camargo ME, Silva GR, Castilho EA, Silveira AC. Inquérito sorológico da prevalência de infecção chagásica no Brasil, 1975/1980. Revista do Instituto de Medicina Tropical de São Paulo 26: 192-204, 1984.

2. Di Primio R. Erros e deficiéncias no diagnóstico de doença de Chagas no Rio Grande do Sul. O problema da provável contaminação por via oral do Trypanosoma cruzi em Teutônia, RS. O Hospital 80: 150-165, 1975. Apud Dias JC. Mecanismos de Transmissão. In: Brener Z, Andrade Z. (ed) Trypanosoma cruzi e doença de Chagas. Ed. Guanabara Koogan, RJ, p. 167-168, 1979.

3. Dorea RC. Doença de Chagas na Amazônia: aspectos epidemiológicos regionais e considerações a propósito de um caso pediátrico. Hiléia Médica, Belem 3: 81-109, 1981.
4. Lainson R, Shaw JJ, Fraiha H, Miles MA, Draper CC. Chagas's disease in the Amazon Basin: I. Trypanosoma cruz $i$ infections in silvatic mammals, triatomine bugs and man, in the State of Para, north Brazil. Transactions of the Royal Society of Tropical Medicine and Hygiene 73: 193-204, 1979.

5. Lainson R, Shaw JJ, Naiff RD. Chagas's disease in the Amazon basin: speculations on transmission "per os". Revista do Instituto de Medicina Tropical de São Paulo 22: 294-297, 1980.

6. Miles MA, Lanham SM, Souza AA, Povoa M. Further enzymic characters of Trypanosoma cruzi and their evaluations for strain identification. Transactions of the Royal Society of Tropical Medicine and Hygiene 74: 221-237, 1980.

7. Miles MA, Povoa MM, Souza AA, Lainson R, Shaw JJ, Ketteridge DS. Chagas's disease in the Amazon Basin: II. The distribution of Trypanosoma cruzi zymodemes 1 and 3 in Para State, north Brazil. Transactions of the Royal Society of Tropical Medicine and Hygiene 75: $667-674,1981$.

8. Miles MA, Souza AA, Povoa M. Chagas's disease in the Amazon Basin. III. Ecotopes of ten triatomine bug species (Hemiptera: Reduviidae) from the vicinity of Belem, Para State, Brazil. Journal of Medical Entomology 18: 266-278, 1981.

9. Nohmi N, Lacerda Jr KG, Salgado A, Mourão O, Santos CAB. Registro do primeiro diagnóstico de doença de Chagas feito no homem no Território Federal do Amapá - Brasil. Boletim do Hospital Escola São Camilo e São Luis 7: 12-19, 1974.

10. Rodrigues BA, Melo GB. Contribuiçào ao estudo da tripanosomiase americana. Memórias do Instituto Oswaldo Cruz 37: 77-90, 1942.

11 Shaw JJ, Lainson R, Fraiha $\mathrm{H}$. Considerações sobre a epidemiologia dos primeiros casos autóctones de doença de Chagas registrados em Belém, Pará, Brasil. Revista de Saúde Pública de São Paulo 3: 153-157, 1969.

12. Silveira FT, Dias MGV, Pardal PP, Lobão AO, Melo GB. Nono caso autóctone de doença de Chagas registrado no estado do Pará, Brasil. (Nota Prévia). Hiléia Médica, Belém 1: 61-62, 1979. 